دراسة البيئة القديمة لتكوين خورمالة بأستخدام متحجرات الاوستراكودا في منطقتي شقلاوة ودهوك، شمالي العراق

صالح خضر خلف محفوظ عبد الله الحديدي

$$
\begin{aligned}
& \text { قسم علوم الأرض } \\
& \text { كلية العلوم } \\
& \text { جامعة الموصل كلئ العلم } \\
& \text { تاريخ الاستلام 2019/8/1 تاريخ القبول 2019/12/31 }
\end{aligned}
$$

\title{
الخلاصة
}

درست البيئة القديمة لتكوين خورمالة باستخدام متحجرات الاوستراكودا لمقطعين من شمال العراق؛ يقع داقع داق الدقطع الاول (مقطع هجران) بالقرب من مدينة شقلاوة، والثاني (مقطع دهوك) بالقرب من مدينة دهوك. من خلال تشخيص متحجرات الاوستراكودا ودراسة ظروف تواجدها البيئي، تم الاستتناج ان بيئة ترسيب تكوين خورمالة كانت تمثل بيئة الرف القاري وبعمق يتراوح ما بين (30 - 200 متر ).

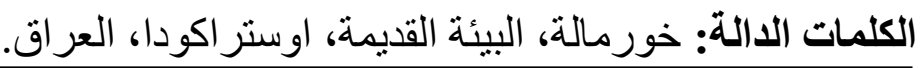

\section{Paleoenvironmental Study of Khurmala Formation by Ostracoda in Shaqlawa and Dohuk area, Northern Iraq}

Mahfoudh Abdulla Al-Hadeedy

Saleh Khder Khalaf

\author{
Dept of Geology \\ College of Science \\ University of Mosul
}

\begin{abstract}
A detail paleoenvironmental study of ostracods fauna to investigated the paleoenvironment of Khurmala Formation for two section Northern Iraq; the first (Hjran section) lies near Shaqlawa city, and the second (Dohuk section) lies near Dohuk city. Based on ostracods study conclude that Khurmala Formation were deposited in shelf environment, about $(30-200)$ meter in depth.

Key word: khurmala, Paleoecology, Ostracoda, Iraq.

\section{المقدمة}

وصف المقطع النموذجي لتكوين خورمالة في بئر (K-114) في منطقة كركوك، مابين الاعماق ( 1176.8) متر من قبل (Bellen, 1953; in Bellen et al., 1959) وبسمك يصل إلى (185 متراً) ويتمثل بتعاقبات من الحجر الجيري الدولومايتي مع بعض السرئيات، فضلا عن الحجر الجيري الناعم التبلور ، ويتداخل هذا التكوين على شكل السنة (Interfingering) مع تكوين كولوش وعمر التكوين هو ( Paleocone- Early
\end{abstract}




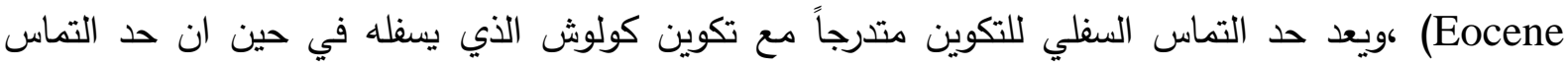

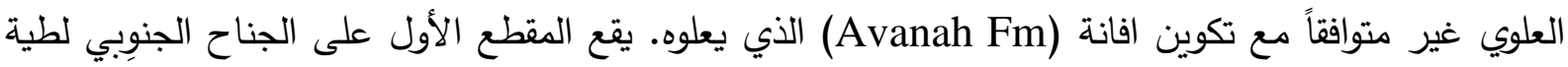

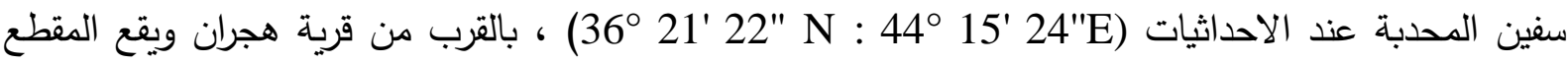
N : 4302 .

تقع منطقة الدراسة تكتونياً ضمن نطاق الرف غير المستقر (Unstable shelf)، تبعاً لتقسيمات

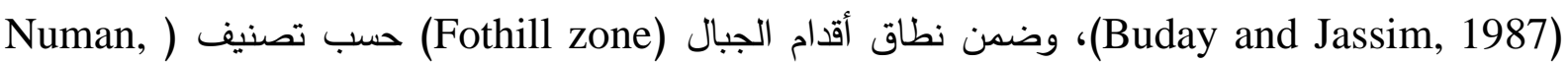

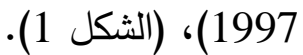

تم جمع (9) نماذج من تكوين خورمالة لمقطع هجران و(23) نموذجاً لمقطع دهوك، حيث يبلغ سمك

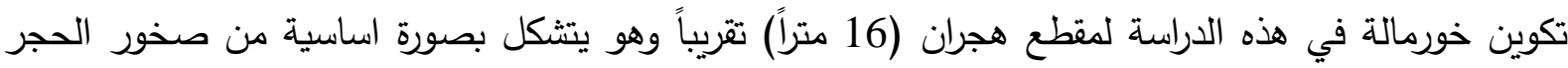

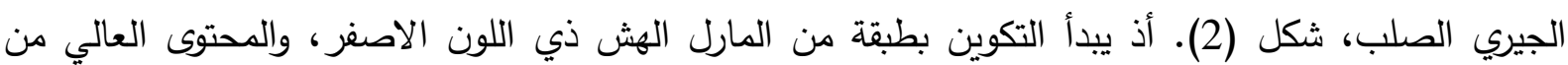

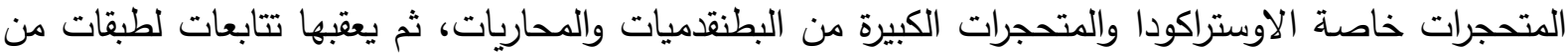
الحجر الجيري الصلب بسك (20-45 سم) لكل طبقة، وتكون متوسطة الصلابة إلى صلبة جدا تحتوي بعضها اصداف واثار لاصداف من المحاريات وبطنية القدم وفتاتات قد تكون من المرجان الشعابي. اما بالنسبة لتكوين خورمالة في مقطع دهوك فانه يظهر بسمك (46 متراً) ويتالف من تعاقبات من الحجر الجيري المارلي، (الثكل 2). وكذلك تعاقبات من الحجر الرملي والسجيل وكذلك طبقات من الحجر الجيري الدولومايتي. إذ يبدا التكوين بتعاقبات لطبقات من الحجر الجيري والحجر الجيري المارلي وقليل من المارل،

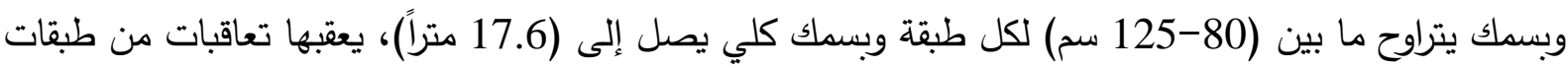

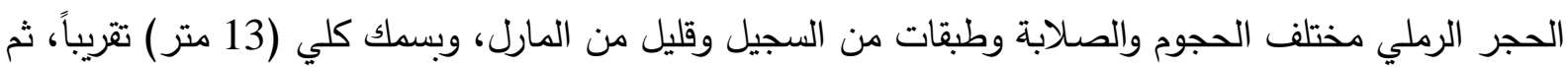
تعاقبات من الحجر الجيري والحجر الجيري المارلي وطبقات من الحجر الدولومايتي وبسمك كلي (12.6 متر) ونهاية المقطع تتمثل بتعاقبات من الحجر الجيري المارلي وبسمك (3 متر) تقريباً.

يعد حد التماس السفلي لتكوين خورمالة في مقطع هجران حداً متدرجاً ومتوافقاً مع تكوين كولوش، ولم يتم ملاحظة دليل يدل على عدم التوافق، في حين ان حد التماس العلوي مع تكوين جركس لم يتم ملاحظتها، بسبب عوامل التعرية التي جرفت الجزء الاسفل من تكوين جركس، ضمن خط سير المقطع وفي المناطق المجاورة القريبة فهو مغطى برواسب حديثة. اما حد التماس السفلي لتكوين خورمالة مع تكوين كولوش في مقطع دهوك،

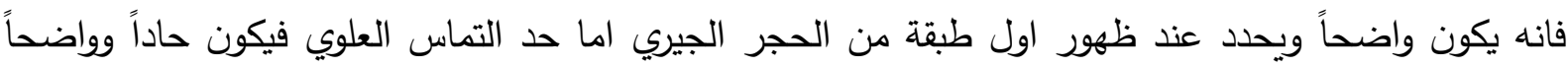
ويحدد عند ظهور اول طبقة فتاتية حمراء تمثل بداية تكوين جركس.

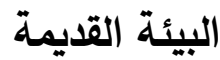

اكتسبت متحجرات الاوستراكودا اهتماماً واسعاً، وذلك من خلال استخدامها كدلائل مهمة في بناء البيئة القديمة، وجاءت هذه الأهمية من خلال حجمها الدقيق وتتوعها الكبير، وقابليتها على المعيشة في مختلف الظروف البيئية، ويتم التعرف على البيئة القديمة باستخدام متحجرات الاوستراكودا عن طريق مقارنتها مع مثيلاتها 
المتواجدة في المياه الحالية، كأفراد دالة فضلا عن الاعتماد على حشود الاوستراكودا المشتركة المعيشة تحت ظروف بيئية محددة، ولقد اقترح (Monostori, 1985) بان من الأهمية والفائدة الكبيرة الاعتماد في دراسة بيئة الاوستراكودا على مستوى الجنس، حيث لوحظ ان أنواع الجنس الواحد تعيش في بيئات متشابهة في اغلب دان الحالات، بينما تقل اهمية استخدام متحجرات الاوستراكودا على مستوى النوع، وذلك لكونها ذات تتوع عالي مرتبط بالظروف البيئية المتغيرة، فهي تعد أنواع محلية أكثر منها عالمية مع ملاحظة وجود انواع دالة لايمكن الاستغناء

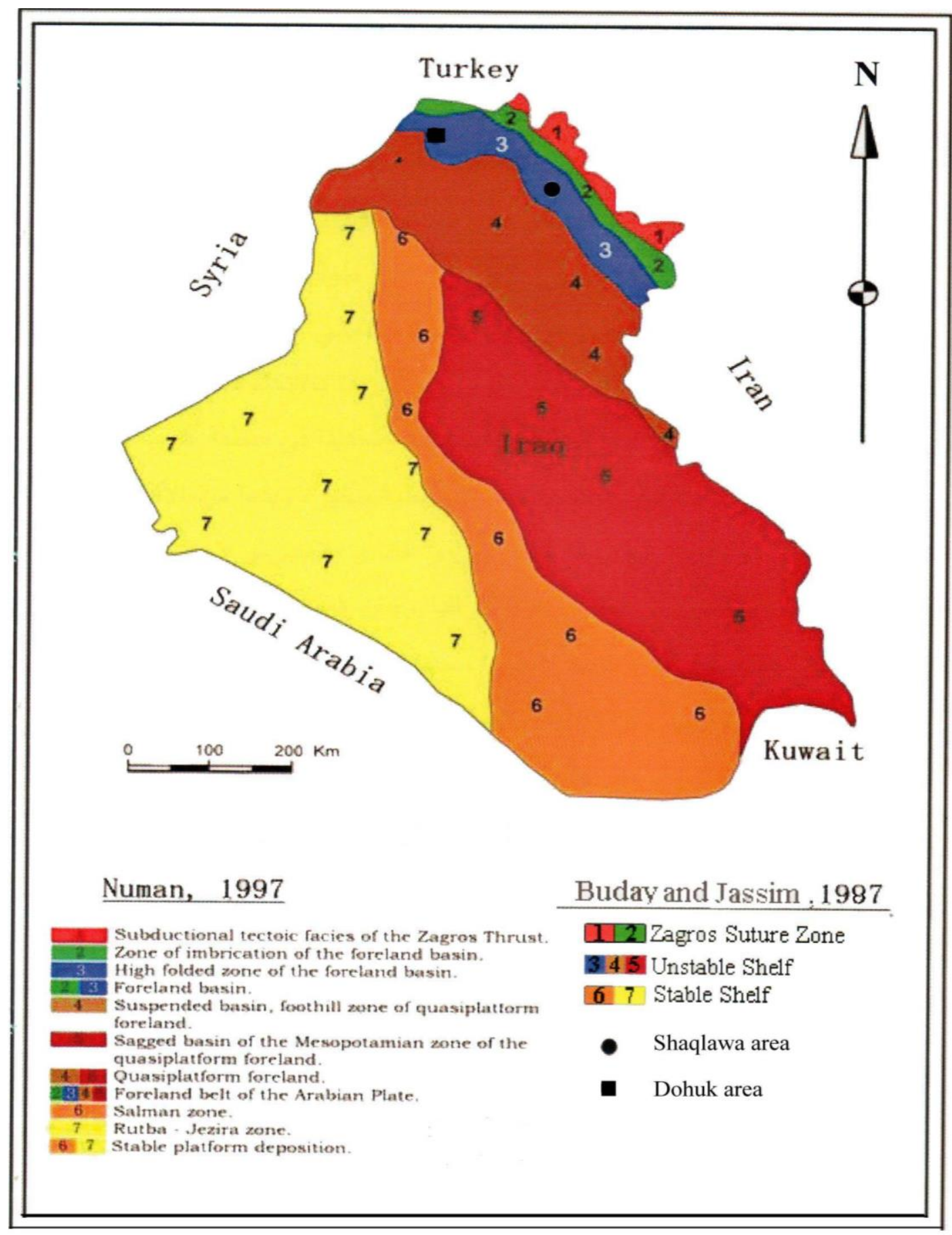

الشكل 1: خارطة التقسيمات التكتونية للعر اق حسب (Buday and Jassim, 1987) و(Numan, 1997) موضحاً عليها منطقتي الدراسة. 


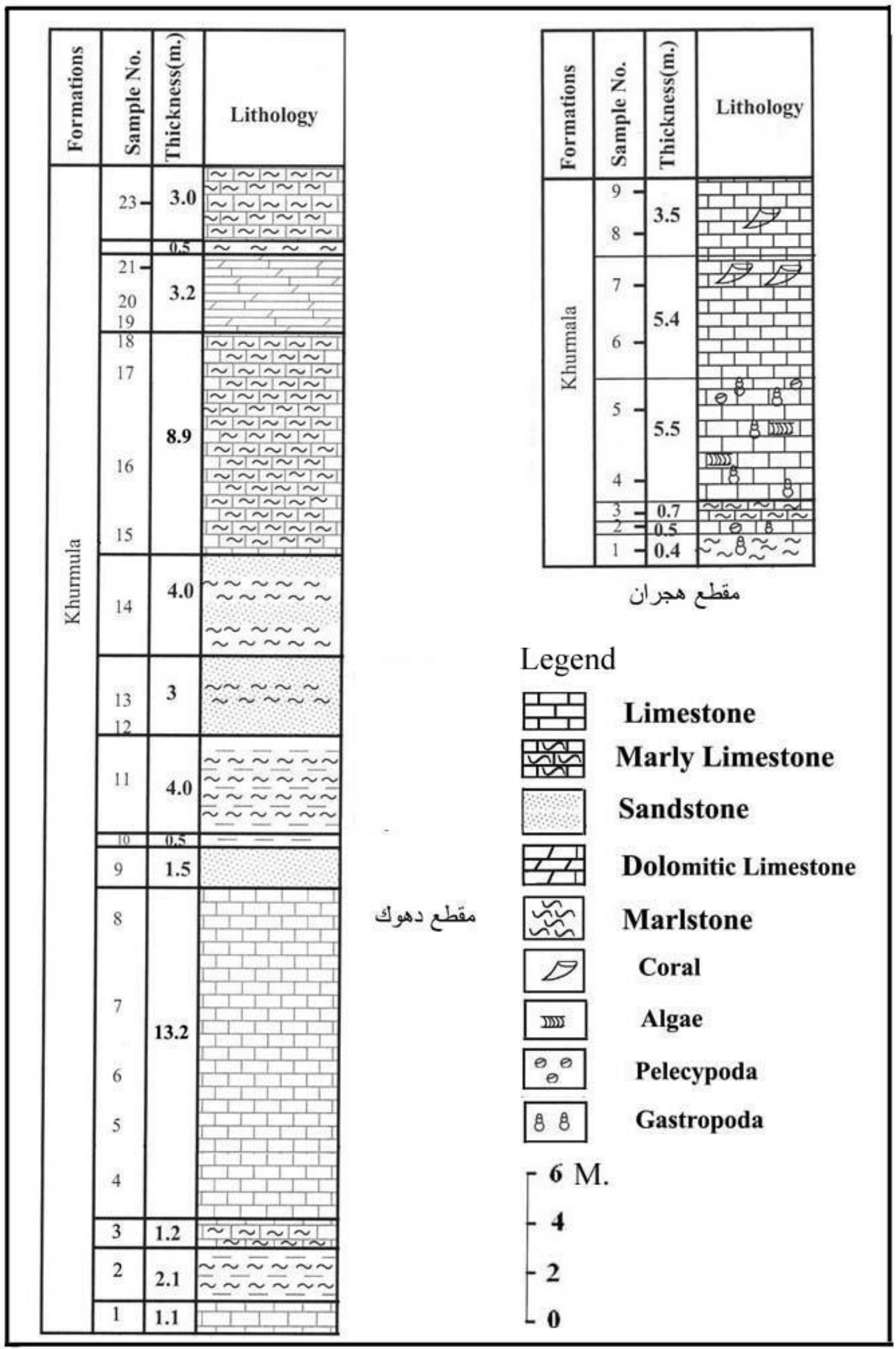

الثكل 2: المقطع الطباقي لتكوين خورمالة لمقطعي هجران ودهوك. 
وضع عدة باحثين تقسيمات مختلفة للأحواض الرسوبية، حسب الاستفادة من ذلك التقسيم، وقد اعتمد في

الدراسة الحالية التقسيم المستخدم من قبل (Flügel, 2010) والمحور عن (Kennet, 1982) والذي يتضمن:

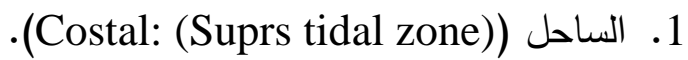

2. الثاطئ (يمتد بين حدي المد والجزر) (Littoral: (Restricted between high and low tide)

\begin{tabular}{|c|c|}
\hline Sub-littoral (Continental Shelf) & 3. الرف القاري \\
\hline Inner shelf $(0-30 \mathrm{~m})$. & 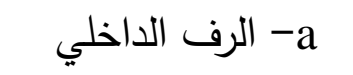 \\
\hline Middle shelf (30 - 100 m.) & b- الرف الأوسط b \\
\hline Outer shelf (100 - 200 m.) & 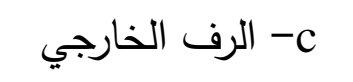 \\
\hline Bathyal (Continental slope) & 4. بيئة المنحدر القاري \\
\hline Inner shelf $(0-30 \mathrm{~m}$. & a - a a a - a الاعلى \\
\hline Middle shelf (30 - 100 m.) & b- b- المنحدر الأوسط \\
\hline Lower slope $(1500-3500$ m.) & 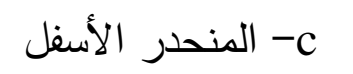 \\
\hline Abyssal (3500 - 5000 m.) & 5 ـ بيئة الابيسال \\
\hline adal (More than $5000 \mathrm{~m}$. & 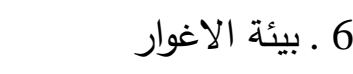 \\
\hline
\end{tabular}

شخص (53) نوعا من الاوستراكودا تعود الى (22) جنسا، من خلال دراسة تتابعات تكوين خورمالة لكلا

المقطعين (plat 1-4) مقسمة بصورة رئيسة إلى أجناس ذات أصداف ملساء تشمل:

Cytherella hastata Neale \&Singh,1985, Cytherella pulchella , Ruggieri ,1967, Cytherella ventroconcava Neale and Singh,1985, Cytherella sp.3 Said 1978, Bairdia beraguaensis Singh and Tewari,1966, Bairdia eocaenica Khalaf and Aziz,1994, Bairdia eocaenica Khalaf and Aziz,1994, Bairdia jammuensis Singh and Tewari,1966, Bairdia ilaroensis Reyment and Reyment,(1959), Bairdia indica Tewari and Tandon,1960, Birdia sayyabi Aziz,2001, Bairdia subdeltoidea(Munster,1830), Neonsidea ilaroides Foster, Swain and Petters,1983, Neonesidea sp.1 Swain,1984, Bairdoppilata gliberti Keij,1957, Bairdoppilata jaswanti Singh and Misra,1968, Bairdoppilata kalakotensis Tewari and Singh,1966, Bairdoppilata rakdiensis Khosla and Pant,1988, Bairdoppilata rajnathi Tewari and Tandon,1960, Bythocypris mianica Tewari and Tandon,1960, Bythocypris aff. sp. Esker,1968, Paracypris rectoventra Sohn,1970, Paracypris wynnei Tewari and Tandon 1960, Argilloecia sp.1 Said,1978, Krithe oryza Neale and Singh,1985, Krithe rutoti Keij,1957, Krithe rutoti Keij,1957, Xestoleberis rupnarayanalurensis Ghuha and Shukla,197, Xestoleberis rupnarayanalurensis Ghuha and Shukla,1973.

أما الاجناس ذات الاصداف التي تمتاز بأنماط زخرفة مختلفة تثمل:

Hermanites palmatus Siddiqui, 1971, Hermanites soliporosa Al-Furaih, 1980, Hermanites sp.2Salahi, 1966, Acanthocythereis cf. conjuncta Al-Furaih, 1980, Acanthocythereis(Canthylocythereis) taqiyeansis Al-Sheikhly,1992, Shizocythere rakhiensis Siddiqui,1981, Shizocythere sorensis Siddiqui,1981, Phalcocythere 
horrescens (Bosquet,1852), Phalcocythere improcera Siddiqui,1971, Phalcocythere(phalcocythere) rectangularis Al-Furaih,1980, Phalcocythere (Prophalcocythere) nullicostata Al-Furaih,1980, Quadracythere orbignyana(Bosquet,1852), Occultocythereis hatraensis Al-Sheikhly,1982, Occultocythereies peristicta Siddiqui,1971, - Anommatocythere cantistrum Al-Furaih and Siddiqui,1981, Anommatocythere cantistrum Al-Furaih and Siddiqui,1981, Anommatocythere laqueta Siddiqui,1971, Schizoptocythere torquata Siddiqui and AlFuraih,1981, Uroleberis iraqensis Khalaf and Aziz,2001, Uroleberis ranikotiana (Latham,1938), Uroleberis Sohni Khosla and Pant,1989, Uroleberis stagnosa AlFuraih,1980, Uroleberis suppsalaensis Carbonnel,Alzouma and Dikouma,1990, Uroleberis suppsalaensis Carbonnel,Alzouma and Dikouma,1990, Buntonia aalijanses Aziz,2002, Buntonia khurmalensis Khalaf and Aziz,2001, Buntonia tatteuliensis(Apostolescu) 1961, Buntonia tatteuliensis(Apostolescu) 1961, Hornibrookella posterisella Al-Furaih,1977, Hornibrookella posterisella AlFuraih,1977

تبين أن الأنواع العائدة للاوستراكودا الملساء تكون قليلة التواجد نسبة الى انواع الاوستراكودا ذات الاصداف

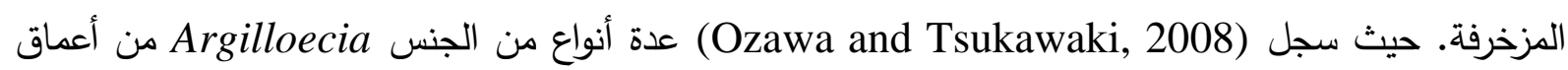

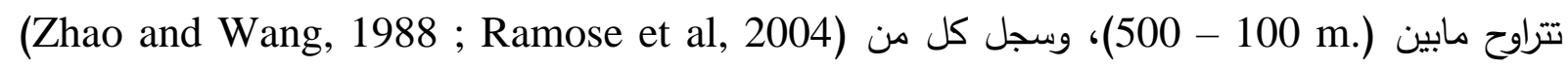

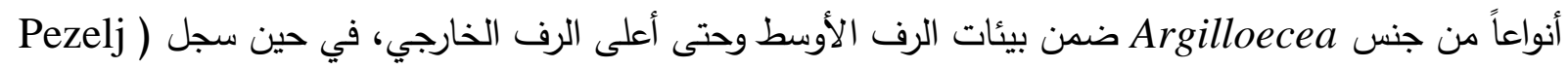

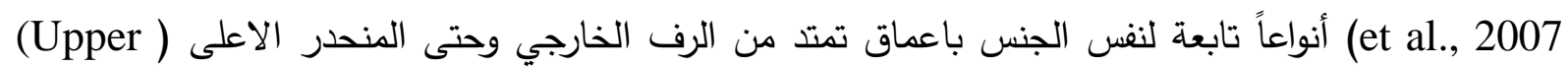

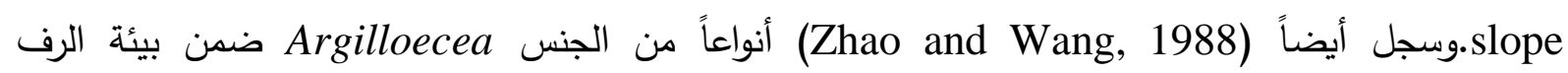

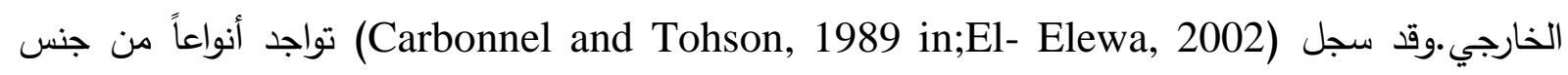
Buntonia

أما بالنسبة للتجمعات الحياتية المشتركة للاوستراكودا فقد سجل (Bergue et al., 2006) في البرازيل

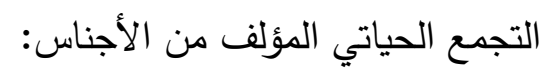

Cytherella, Bairdoppilata, Bythocypris, Krithe, and Xestoleberis.

ضمن بيئات المنحدر الأعلى وبداية المنحدر الأوسط، وسجل (Shahin, 2005) التجمع الحياتي Bairdia, Bairdoppilata, Xestoleberis, Krithe, Paracypris and Cytherella مثل Buntonia ضمن بيئات الرف الأوسط و الخارجي وسجل (Shahin et al., 2008) الحشد الحياتي Cytherella, Krithe, Buntonia, Argilloecea and Cytheridea Cytherella, يصل الى (200 متر)، وسجل (Kuznetsova and Doborova, 1995) التجمع الحياتي Bairdia and Microcypris

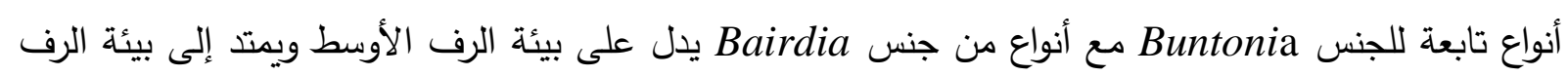
الخارجي، وذكر (Monostori, 1985) أن التجمع المؤلف من الاجناس: Cytherella, Bairdia) and Occultocythereis Bairdoppilata, Xestoleberis, Paracypris وبذلك يتم الاعتماد على الأجناس المشتركة معها في تحديد البيئة القديمة، فعند تواجدها مع الأنواع ذات الدروع 
السميكة والزخرفة القوية والعقد العينية البارزة تكون قرببة من البيئات الضحلة، ومع نقصان هذه الأجناس والزيادة في نسبة الأصداف الملساء فيكون هنالك زيادة في العمق حتى تصل إلى بيئات المنحدر القاري.

أما بالنسبة للأجناس ذات الأصداف المزخرفة فقد سجل (Siddiqui and Al-Furaih, 1981) أنواعاً

من الجنسين Schizeptocythere and Schizocythere من المياه الضحلة في السعودية. وسجل كل من (Al-Furaih and Siddiqui, 1981, Brouwers and Fatmi,1992, Al-Furaih, 1980, Nazik, 1993 and Monostori, 1985)

Sissingh, ) وسجل تواجده من قبل (Athersuch, 1979) من الأعماق الضحلة الحالية وسجل تواجده أيضاً متهات 1972) من الأعماق الضحلة التي لا تزيد عن (50 متراً) في جنوب بحر ايجة. وقد سجل ( Van (15) (Morkhoven, 1963 و (Nazik, 1993) أنواعاً من الجنس Quadracythere) ضمن الأعماق الضحلة

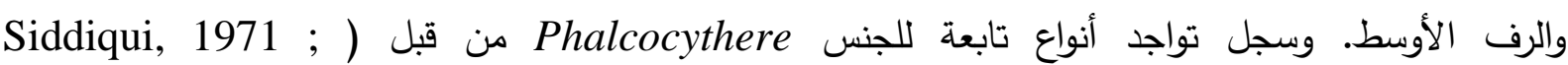
(Monostori, 1985 في بيئات ضحلة نيريتية وبيئات الرف الداخلي والأوسط.

أما بالنسبة لأنواع الجنس Xestoleberis فانه من الأجناس ذات الزخرفة الملساء ولكنه يتواجد في البيئات Van Morkhoven, 1963 ; الضحلة التي تتواجد فيها الأجناس ذات الزخرفة القوية حيث سجل كل من لونداع (Tunogulu and تواجده ضمن الأعماق الضحلة من الرف القاري، وسجل تواجده كل من (Nazik, 1993) ( Unal, 2001 ; Caus et al., 2002 and Opreanu, 2004) الأوسط وبعمق لا يتجاوز (50 متراً)، وسجله (Athersuch, 1979) ضمن الأعماق البحرية الضحلة الحالية للبحر الأبيض المتوسط وذكر (Zhao and Wang, 1988) أن أنواع هذا الجنس تعيش بأعماق ضحلة وتمتد إلى الرف الخارجي، وسجلت أفراد تابعة للجنس Uroleberis من قبل كلا من ; Monostori, 1985 and Zhao and Wang, 1988) إلى نهاية الرف الخارجي.

أما بالنسبة للتجمعات الحياتية لأجناس الاوستراكودا فقد أثار (Keen et al., 1994) إلى أن الأجناس Cytherella, Bairdia, Bairdoppilata, Xestoleberis, Paracypris and Occultocythereis Hermanites, Quadracythere, Schizoythere ترافقها مع أجناس ذات زخرفة قوية ، وعيون متطورة مثل فيدل على أنها ذات بيئة تمثل الرف القاري وهي نموذجية لبيئات الرف الأوسط وبأعماق تتراوح ما بين عدة أمتار إلى (50 متر) تقريباً. وذكر (Ozawa and Kamiya, 2005) أن التجمع المؤلف من:Acanthocythereis, Argilloecia,

Krithe, Neonsidea, Schizocythere, and xestoleberis , وسجل يمثل بيئات الرف الأوسط

Cytherella, Krithe, Argilloecio, and Buntonia الحثد الحياتي المؤلف من (Rogl et al., 2008) مع أجناس ذات زخرفة قوية مثل:

Quadracythere, Hornibrookella, Hermanites and Acanthocythereis

$$
\text { تدل على بيئة الرف الخارجي. }
$$




\section{الاستنتاجات}

شخص (53) نوعا من الاوستراكودا تعود الى(22) جنسا واظهرت افراد هذه الانواع مختلف انواع الزخرفة

من الزخرفة الملساء وحتى الزخرفة الثديدة ومن خلال مناقثة طبيعة معيشتها وظروف تواجدها وانتشارها البيئي

تم استتاج ان بيئة الترسيب لتكوين خورمالة للمقطعين تقع ضمن بيئات ضحلة متمثلة بالرف الداخلي وحتى نهاية الرف الخارجي وبعمق يتراوح ما بين (200 m- 200).

\section{REFERENCES}

Al-Furaih, A.A.F., 1980. Upper Cretaceous and Lower Tertiary Ostracoda (Superfamily Cytheracea) from Saudi Arabia. Univ. Libraries. Univ. Riyadh. Saudi Arabia, 211 P.

Athersuch, J. 1979. The ecology and distribution of the littoral ostracoda. Cypris.Jour. of Nat. Hist., Vol. 13, PP. 135-160.

Bellen Van, R. C., Dunnington, H. V., Wetzel, R. and Mortan, D. M., 1959. Lexique Stratigraphique International, Vol.3, Asie, Fascicule, 10a-Iraq Cent. Nat. Sci., Paris, $333 \mathrm{p}$.

Bergue,C.T., Costa,K.B.,Dwyer, G. and Moura,C.A.,2006 .Bathyal ostracode diversity in the Santos Basin, Brazilian SouthEast Margini response to Late Quaternary climate changes. Rev.bras. Paleon. Vol.9, No.2, PP.201-210.

Brouwers, E. and Fatmi,S.F. ,1992 . Late Paleocene-Early Eocene ostracode emblages from core UAJ-1, Meting-Jhimpir coal field,Sindh province, Pakistan. open - file report ,No.92-221,PP.1-46.

Buday, T., and Jassim, S.Z., 1987. The Regional Geology of Iraq. Vol. 2, Tectonism, Magmatism and Metamorphism, Baghdad, $352 \mathrm{P}$.

Caus, E., Tambareau, Y., Colin,J., Aguilar, M., Bernaus, J., and Brusset, S.,2002. Upper Creataceous microfauna of the Formation San Luis Potosi, NE Mexico. Biostratigraphical, palaeoecological and palaeogeographical significance. Revista Mexicana de Cienicas Geologicas, Vol. 19, No. 2, PP. 137-144.

El-Elewa, A.M.T., 2002. Paleobiogeography of Maastrichtian to Early Eocene ostracoda of North and West Africa and the Middle East.Micropal.Vol.48, No.4, PP.391-398.

Flügel, E., 2010. Microfacies of carbonate rocks. Springer-Verlag Berlin Heidelberg, $984 \mathrm{P}$.

Keen, M.C., Al-Sheikly,S.S.J. , Elsogher,A ,and Gammudi,A.M., 1994. Tertiary ostracods of North Africa and Middle East. In: Simmons, M.D., (ed.), Micropal. and hydrocarbon exploration in the Middle East.

Kuznetsova, K.I. and Dobrova, M.R., 1995. Enemic and cosmopolitan assemblages of foraminifers and ostracods from the Middle Jurassic basins of Syria .Strrat. and Geological Correlation, Vol.3.

Monostori, M., 1985. Eocene ostracoda from the Dorog Basin (Northern Transdanubia, Hungary) Akademia Kaido Budapest, PP. 1-214. 
Nazik, A., 1993. Macropaleontological (ostracoda and foraminifera) Investigation of Tertiary Sequence of Darendes basin. Geol. Bull. Turkey, Vol. 36, PP. 13-36.

Neal, T.W.,1988. Ostracods and paleosalinity reconstruction. In: De Decker, P., Colin,j.p. and Peypouquet,J.P. (eds.) ;Ostracoda in the Earth Sciences. Elsevier, Amsterdam, Oxford, New York, Tokyo. PP.126-155.

Numan, N. M. S., 1997. A plate tectonic scenario for the Phanerozoic succession in Iraq, Iraqi Geol. Jour., Vol. 30, No. 2, PP. 85-110.

Opereanu, P., 2004. Some data on the recent ostracoda fauna from the continental shelf of the Black Sea in the Crimea and Sinop areas. Geo-Eco- Marina, No. 910, 2003-2004. PP.

Ozawa, H. and Kamiya, T., 2005. Ecological analysis of benthic ostracods in the Northern Japan Sea, based on water properties of modern habitatus and Late Cenozoic fossil record. Marine Micropal., Vol. 55, PP. 255-276.

Ozawa, H. and Tsukawaki, Sh., 2008. Preliminary report on modern ostracods in surface sediment sample sollected during R.V. Tansei-maru Cruse K T4-20 in Southwestern Okhotsk Sea and the Northeastern Japan Sea off Hokkaido, North Japan. Annals of Research Institute of the Japan Region, Vol. 39, PP. 31-48.

Pezelj, D., Sremac,J. and Sokac, A., 2007. Palaeoecology of the Late Badenian foraminifera and ostracoda from the SW central Paratethys (Medvednica mt.) Coroatia. Geologia Croatica, Vol. 60, No. 2, PP. 139-150.

Ramos, M.I.F., Watley, R.C. and Coimbra, J.C., 2004. Sub-recent marine ostracoda (Pontocyprididae and Bairdiidae) from the Southern Brazilian continental shelf. Revista Brazileira Pal. Vol.7, No.3, PP. 311-318.

Rogl, F., Coric, S. Harzhauser, M., Kroh, A. Schultz, O., Wessely, G. and Zorn, I. 2008. The Middle Miocene Badenian Stratotype at Baden-Sooss, Geologica Carpathica, October, Vol. 59, No. 5, PP. 367-374.

Shahin, A., 2005. Tertiary ostracods of Gebel Withr, Southwestern Sinai, Egypt: palaeontology. Biostratigraphy and palaeobiogeography. Revue de Paleobiologie, Geneve, Vol. 24, No. 2, PP. 749-779.

Shahin, A., El-Halaby, O. and El. Baz, S., 2008. Middle Eocene ostracodes of the Qattamiya area, Northwest Eastern Desert, Egypt: Systematics, Biostratigraphy and Paleobiogeography. Revue de paleobiologie, Geneve, vol. 27, No. 1, PP. 123-157.

Siddiqui, Q. A. 1971. Early Tertiary ostracoda of the Family Trachyleberididae from West Pakistan, Bull, Br. Mus. Nat. Hist. Geol. Suppl. Vol. 9, PP. 1-98.

Siddiqui, Q. A. and Al-Furaih, A. A. F., 1981. A new Trachyleberid ostracod genus from the Early Tertiary of western Asia, Pal., Vol. 24, Part 4, PP. 877- 890.

Tunoglu, C. and Unal, A., 2001. Ostracoda biostratigraphy of Pannonian-Pontian sequence of Gelibolu Peninsula, NW Turkey Geol. Bull. of Turkey, Vol. 44, No. 1, PP 15-25. 
Van Morkhoven, F.P.C., 1963. Post-Paleozoic Ostracoda. Elsevier Pub. Comp., Amesterdam, Part, 2, $478 \mathrm{P}$.

Zhao, Q. and Wang, P., 1988. Distribution of modern ostracoda in the shelf seas off China. In: Hanai, T., Ikeya, N. and Ishizaki, K. (eds.), Evolutionary Biology of Ostracoda. PP. 805-819, Elsevier.

\section{Explanations of Plate (1)}

\section{scale: 1 bar $=100 \mu \mathrm{m}$}

Fig.1- Cytherella hastata Neale \&Singh, 1985. Carapace (Mo.T.Kh.2) External left lateral view Khurmala Fm.,Kh.1 ,Hjran section

Fig.2- Cytherella pulchella Ruggieri ,1967. Carapace (Mo.T.Kh.4) External left lateral view Khurmala Fm.,Kh.2 ,Hjran section

Fig.3- Cytherella ventroconcava Neale and Singh, 1985.Carapace (Mo.T.Kh.5) External left lateral view Khurmala Fm., Kh.1, Hjran section

Fig.4- Cytherella sp.3 Said 1978. Carapace (Mo.T.Kh.6) External left lateral view Khurmala Fm.,Kh.6 ,Dohuk section

Fig.5- Bairdia beraguaensis Singh and Tewari, 1966.Carapace (Mo.T.Kh.8) External right lateral view Khurmala Fm.,Kh.1 ,Hjran section

Fig.6-Bairdia eocaenica Khalaf and Aziz, 1994. Carapace (Mo.T.Kh.9) External right lateral view Khurmala Fm.,Kh.1 ,Hjran section

Fig.7- Bairdia jammuensis Singh and Tewari, 1966. Carapace (Mo.T.Kh.10)

External right lateral view Khurmala Fm., Kh.4 ,Dohuk section

Fig.8- Bairdia ilaroensis Reyment and Reyment,(1959). Carapace (Mo.T.Kh.12) External right lateral view Khurmala Fm., Kh.2 ,Hjran section

Fig.9-Bairdia indica Tewari and Tandon, 1960. Carapace (Mo.T.Kh.13 External right lateral view Khurmala Fm., Kh.8 ,Dohuk section

Fig.10- Birdia sayyabi Aziz, 2001. Carapace (Mo.T.Kh.15) External right lateral view Khurmala Fm., Kh.2 ,Hjran section

Fig.11- Bairdia subdeltoidea(Munster,1830). Carapace (Mo.T.Kh.16) External right lateral view Khurmala Fm.,Kh.1 ,Hjran section

Fig.12- Neonsidea ilaroides Foster,Swain and Petters,1983. Carapace (Mo.T.Kh.17) External right lateral view Khurmala Fm.,Kh.2 ,Hjran section

Fig.13- Neonesidea sp.1 Swain, 1984. Carapace (Mo.T.Kh.18) External right lateral view Khurmala Fm., Kh.1 ,Hjran section 
Fig.14- Bairdoppilata gliberti Keij, 1957. Carapace (Mo.T.Kh.19) External right lateral view Khurmala Fm.,Kh.1 ,Hjran section

Fig.15- Bairdoppilata jaswanti Singh and Misra, 1968. Carapace (Mo.T.Kh.20)

External right lateral view Khurmala Fm., Kh.5,Dohuk section

\section{Explanations of Plate (2)}

Fig.1- Bairdoppilata kalakotensis Tewari and Singh, 1966. Carapace (Mo.T.Kh.21)

Fig. 2- Bairdoppilata rakdiensis Khosla and Pant,1988. Carapace (Mo.T.Kh.22)

External right lateral view Khurmala Fm., Kh.1 ,Hjran section

Fig.3- Bairdoppilata rajnathi Tewari and Tandon, 1960. Carapace (Mo.T.Kh.23)

External right lateral view Khurmala Fm., Kh.11,Dohuk section

Fig.4- Bythocypris mianica Tewari and Tandon, 1960. Carapace (Mo.T.Kh.25) External right lateral view Khurmala Fm., Kh.2 ,Hjran section

Fig.5- Bythocypris aff. sp. Esker,1968. Carapace (Mo.T.Kh.27) External right lateral view Khurmala Fm., Kh.1 ,Hjran section

Fig.6- Paracypris rectoventra Sohn, 1970. Carapace (Mo.T.Kh.29) External right lateral view Khurmala Fm., Kh.11, Dohuk section

Fig.7- Paracypris wynnei Tewari and Tandon 1960. Carapace (Mo.T.Kh.30)

External right lateral view Khurmala Fm., Kh.1 ,Hjran section

Fig.8- Argilloecia sp.1 Said, 1978. Carapace (Mo.T.Kh.35) External right lateral view Khurmala Fm., Kh.1 ,Hjran section

Fig.9- Shizocythere rakhiensis Siddiqui, 1981. Carapace (Mo.T.Kh.36) External left lateral view Khurmala Fm., Kh.1,Hjran section

Fig.10- Shizocythere sorensis Siddiqui, 1981. Carapace (Mo.T.Kh.37) External right lateral view Khurmala Fm., Kh.2 ,Hjran section

Fig.11- Krithe oryza Neale and Singh, 1985. Carapace (Mo.T.Kh.40) External right lateral view Khurmala Fm., Kh.4, Dohuk section

Fig.12- Krithe rutoti Keij, 1957. Carapace (Mo.T.Kh.41) External right lateral view Khurmala Fm., Kh.2 ,Hjran section

Fig.13- Krithe rutoti Keij, 1957. Carapace (Mo.T.Kh.42) External right lateral view Khurmala Fm., Kh.2 ,Hjran section

Fig.14- Dentokrithe autochthona(Lyubimova and Guha),1960. Carapace

(Mo.T.Kh.43) External right lateral view Khurmala Fm., Kh.5 ,Dohuk section

Fig.15- Occultocythereis hatraensis Al-Sheikhly,1982. Carapace (Mo.T.Kh.44)

External right lateral view Khurmala Fm., Kh.2 ,Hjran section 


\section{Explanations of Plate (3)}

Fig.1- Occultocythereies peristicta Siddiqui,1971. Carapace (Mo.T.Kh.46) External right lateral view Khurmala Fm., Kh.3, Dohuk section

Fig.2- Schizoptocythere torquata Siddiqui and Al-Furaih, 1981. Carapace (Mo.T.Kh.48) External right lateral view Khurmala Fm., Kh.2, Hjran section

Fig.3- Acanthocythereis cf. conjuncta Al-Furaih, 1980. Carapace (Mo.T.Kh.49) External left lateral view Khurmala Fm., Kh.14, Dohuk section

Fig.4- Acanthocythereis(Canthylocythereis) taqiyeansis Al-Sheikhly,1992. Carapace (Mo.T.Kh.50) External left lateral view Khurmala Fm., Kh.2 ,Hjran section

Fig.5- Phalcocythere horrescens (Bosquet, 1852). Carapace (Mo.T.Kh.51) External right lateral view Khurmala Fm., Kh.2 ,Hjran section

Fig.6- Phalcocythere improcera Siddiqui, 1971. Carapace (Mo.T.Kh.52) External left lateral view Khurmala Fm., Kh.9, Dohuk section

Fig.7- Phalcocythere(phalcocythere) rectangularis Al-Furaih,1980. Carapace (Mo.T.Kh.53) External right lateral view Khurmala Fm., Kh.1, Hjran section

Fig.8- Phalcocythere (Prophalcocythere) nullicostata Al-Furaih, 1980.Carapace (Mo.T.Kh.54) External right lateral view Khurmala Fm., Kh.11, Dohuk section

Fig.9- Paragrenocythere gladius Al-Furaih, 1980. Carapace (Mo.T.Kh.55) External left lateral view Khurmala Fm., Kh.5, Dohuk section

Fig.10- Anommatocythere cantistrum Al-Furaih and Siddiqui, 1981. Carapace (Mo.T.Kh.58) External left lateral view Khurmala Fm., Kh.1, Hjran section

Fig.11- Anommatocythere cantistrum Al-Furaih and Siddiqui, 1981. Carapace (Mo.T.Kh.59) External left lateral view Khurmala Fm., Kh.1, Hjran section

Fig.12- Anommatocythere laqueta Siddiqui, 1971. Carapace (Mo.T.Kh.60) External right lateral view Khurmala Fm., Kh.5, Dohuk section

Fig.13- Buntonia aalijanses Aziz,2002. Carapace (Mo.T.Kh.61) External left lateral view Khurmala Fm., Kh.3, Dohuk section

Fig.14- Buntonia khurmalensis Khalaf and Aziz, 2001.Carapace (Mo.T.Kh.62) Externalright lateral view Khurmala Fm., Kh.13, Dohuk section

Fig.15- Buntonia tatteuliensis(Apostolescu) 1961. Carapace (Mo.T.Kh.63) External left lateral view Khurmala Fm., Kh.3, Dohuk section 


\section{Explanations of Plate (4)}

Fig.1- Buntonia tatteuliensis(Apostolescu) 1961. Carapace (Mo.T.Kh.64) External right lateral view Khurmala Fm., Kh.3, Dohuk section

Fig.2- Hermanites palmatus Siddiqui ,1971. Carapace (Mo.T.Kh.65) External left lateral view Khurmala Fm., Kh.1, Hjran section

Fig.3- Hermanites soliporosa Al-Furaih, 1980. Carapace (Mo.T.Kh.66) External right lateral view Khurmala Fm., Kh.32, Hjran section

Fig.4- Hermanites sp.2 Salahi, 1966. Carapace (Mo.T.Kh.67) External left lateral view Khurmala Fm., Kh.9 , Dohuk section

Fig.5- Quadracythere orbignyana(Bosquet,1852). Carapace (Mo.T.Kh.68) External right lateral view Khurmala Fm., Kh.1, Hjran section

Fig.6- Hornibrookella posterisella Al-Furaih,1977.Carapace (Mo.T.Kh.69) External left lateral view Khurmala Fm., Kh.1 , Hjran section

Fig.7- Hornibrookella posterisella Al-Furaih,1977.Carapace (Mo.T.Kh.70) External left lateral view Khurmala Fm., Kh.1, Hjran section

Fig.8- Xestoleberis rupnarayanalurensis Ghuha and Shukla, 1973. Carapace (Mo.T.Kh.74) Externalright lateral view Khurmala Fm., Kh.9, Dohuk section

Fig. 9- Xestoleberis rupnarayanalurensis Ghuha and Shukla, 1973.Carapace (Mo.T.Kh.74) External right lateral view Khurmala Fm., Kh.9 ,Dohuk section

Fig.10- Uroleberis iraqensis Khalaf and Aziz, 2001.Carapace (Mo.T.Kh.75) External right lateral view Khurmala Fm., Kh.1 ,Hjran section

Fig.11- Uroleberis ranikotiana (Latham, 1938). Carapace (Mo.T.Kh.76) External carapace view Khurmala Fm., Kh.1 ,Hjran section

Fig.12- Uroleberis Sohni Khosla and Pant,1989. Carapace (Mo.T.Kh.77) External carapace view Khurmala Fm., Kh.1 ,Dohuk section

Fig.13- Uroleberis stagnosa Al-Furaih, 1980. Carapace (Mo.T.Kh.78) External right lateral view Khurmala Fm., Kh.8 ,Dohuk section

Fig.14- Uroleberis suppsalaensis Carbonnel,Alzouma and Dikouma,1990. Carapace (Mo.T.Kh.79) External right lateral view Khurmala Fm., Kh.14, Dohuk section

Fig.15- Uroleberis suppsalaensis Carbonnel,Alzouma and Dikouma,1990. Carapace (Mo.T.Kh.80) External right lateral view Khurmala Fm., Kh.14, Dohuk section 


$$
\text { PLATE (1) }
$$
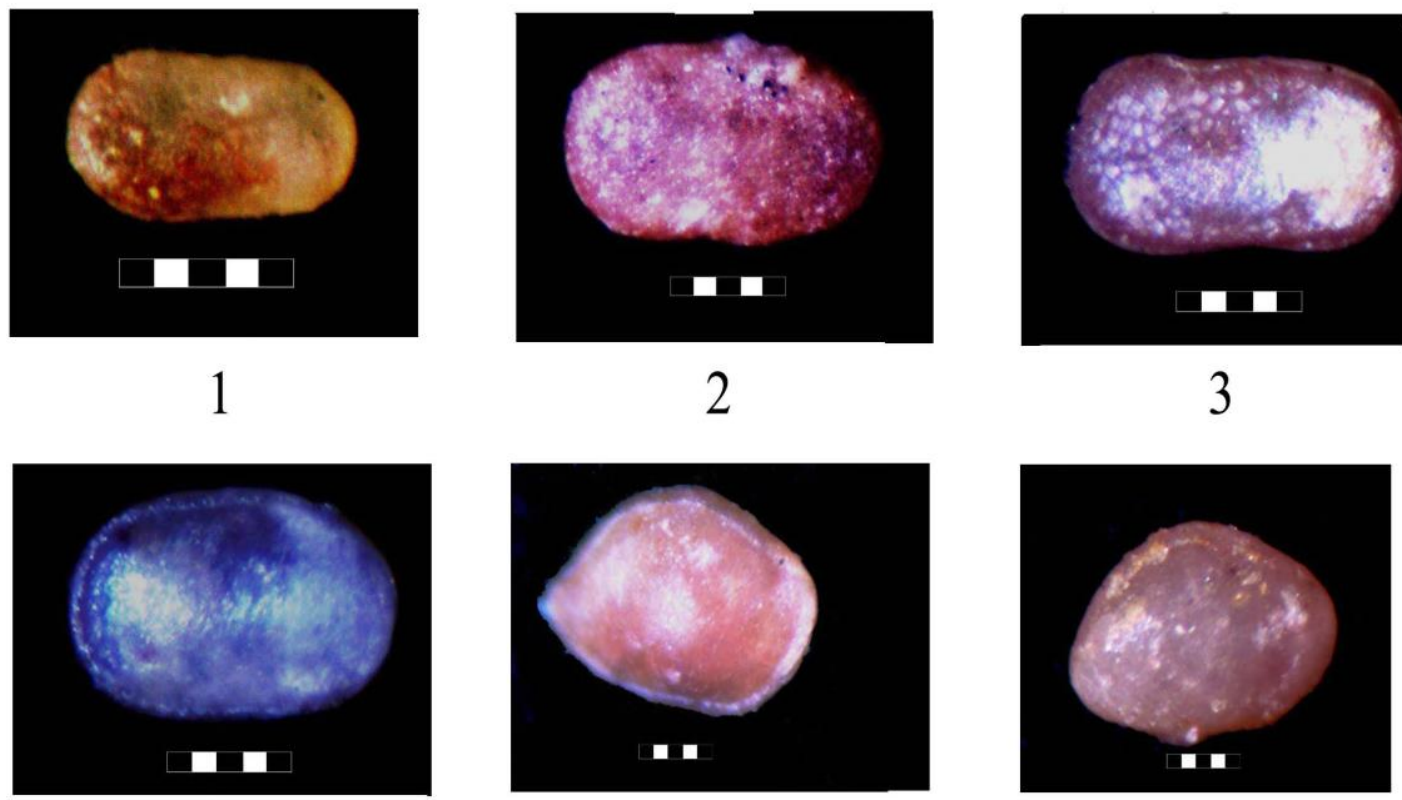

4

5

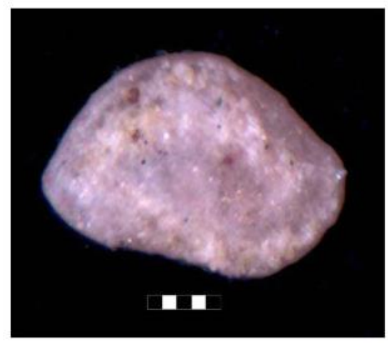

7
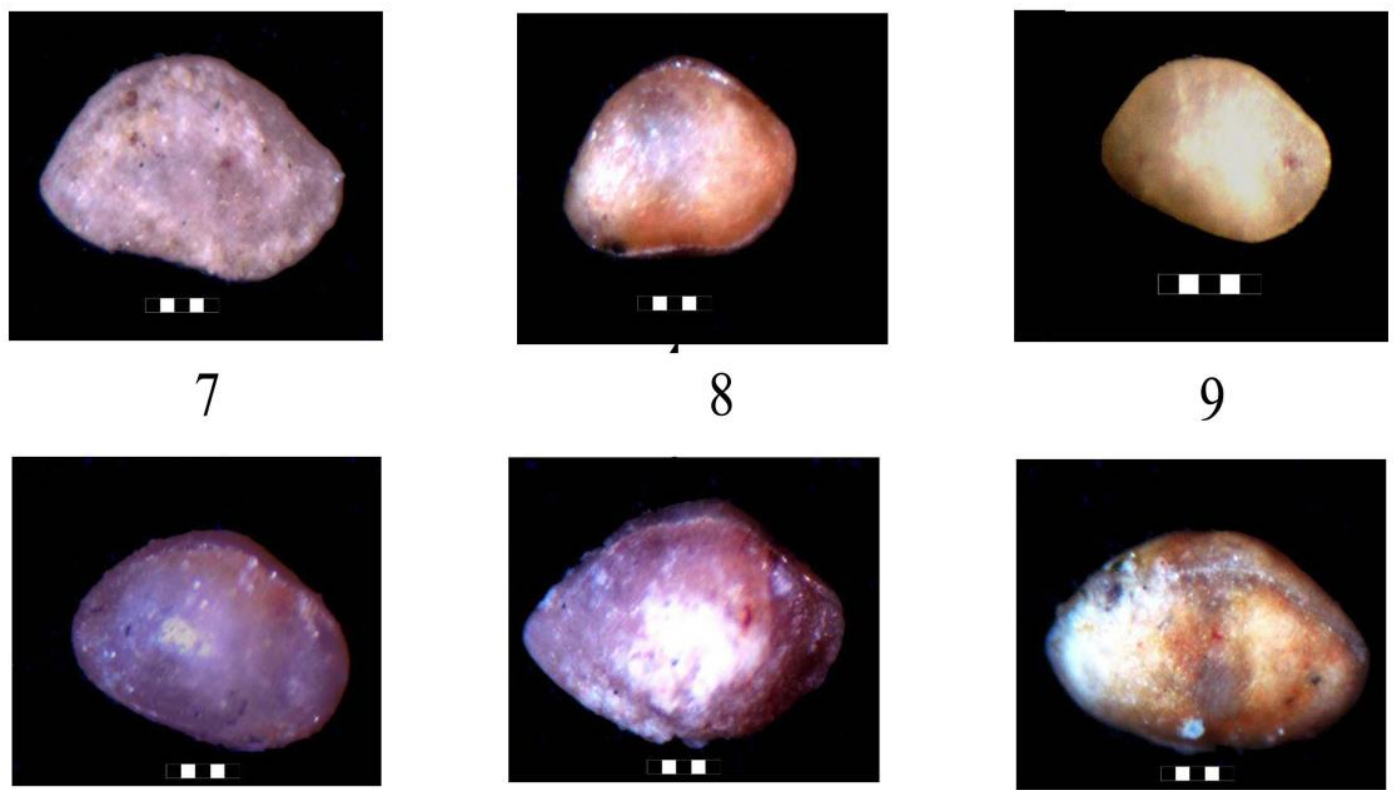

10

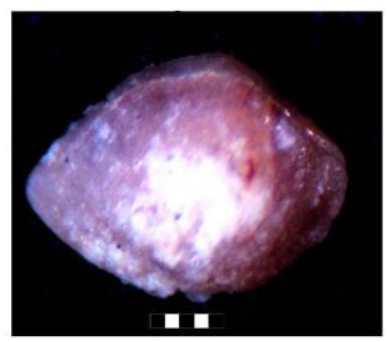

11

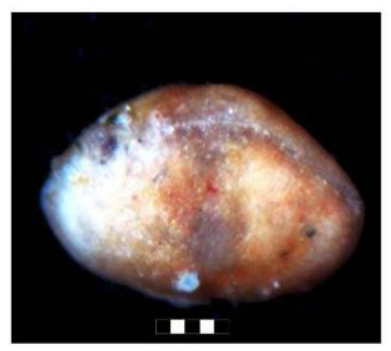

12

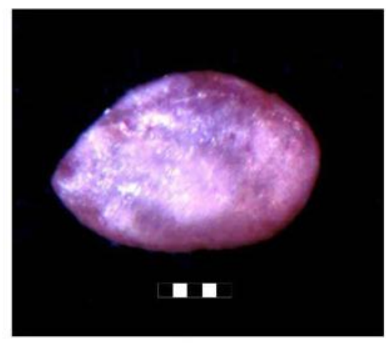

13

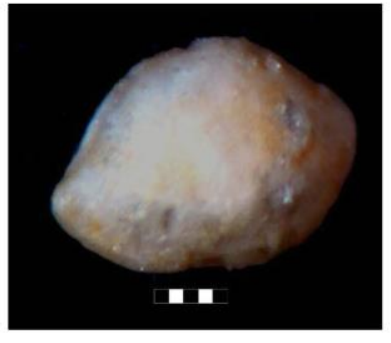

14

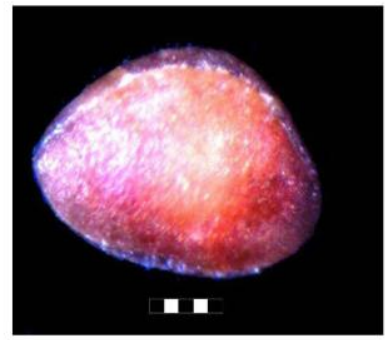

15 
PLATE (2)

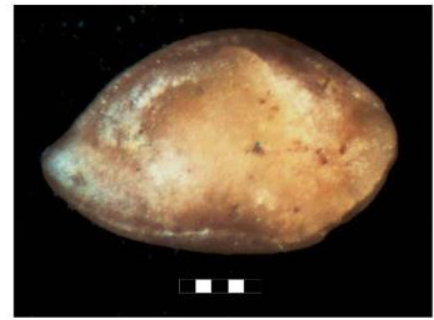

1

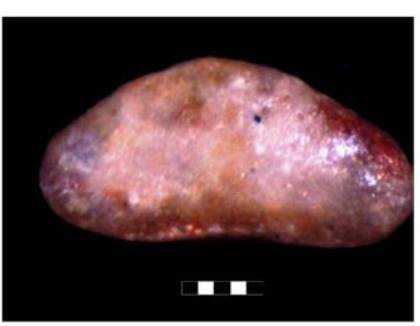

4

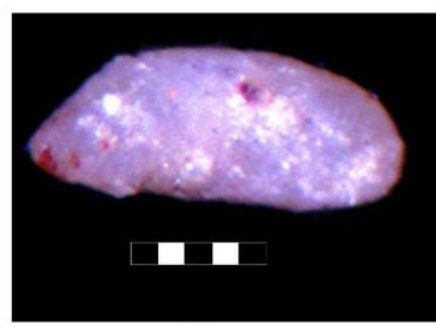

7

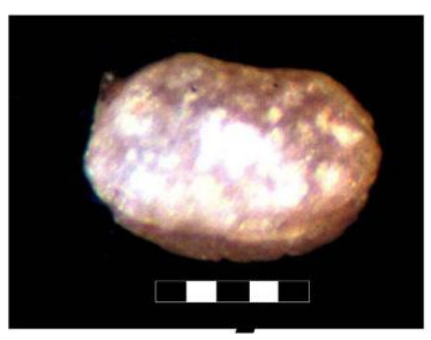

10

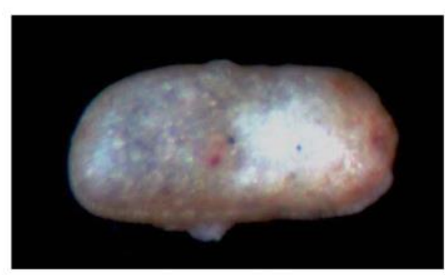

13

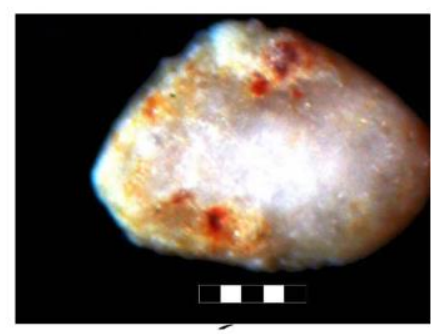

2

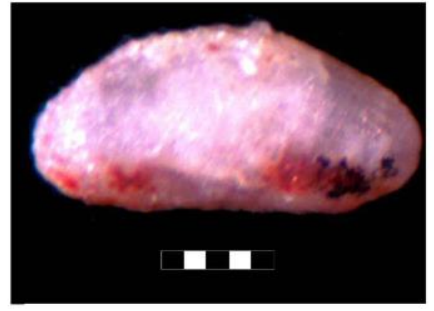

5

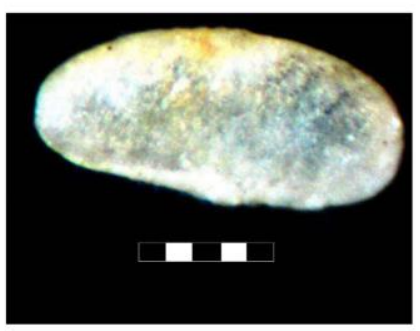

8

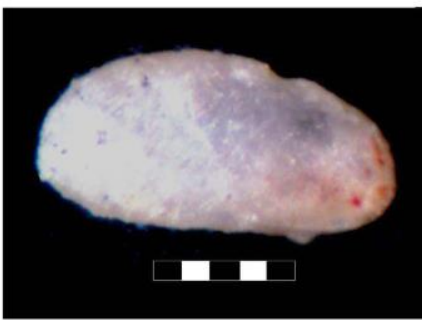

11

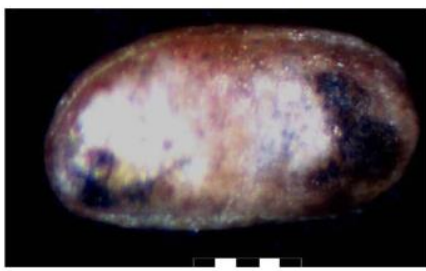

14

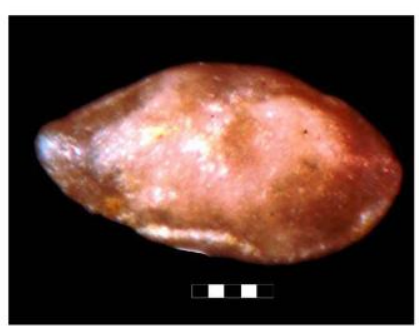

3
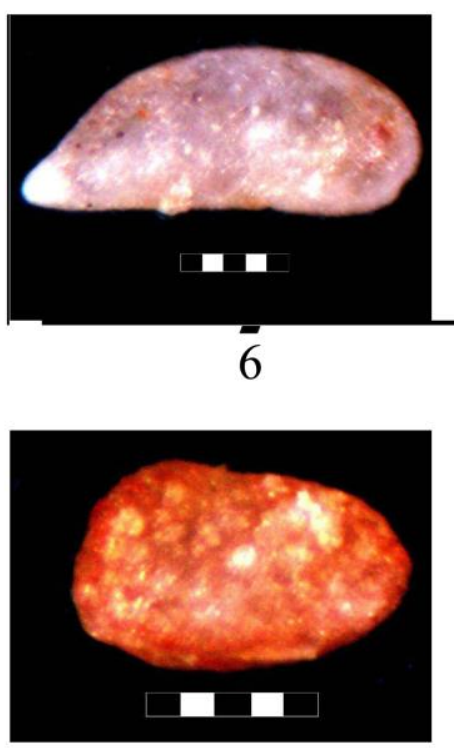

9

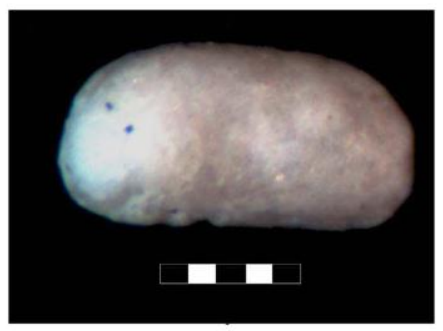

12

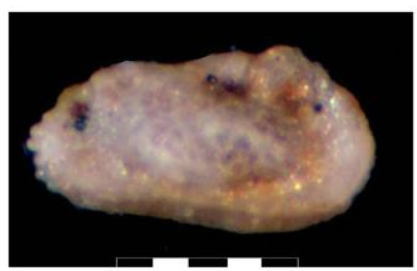

15 
PLATE (3)
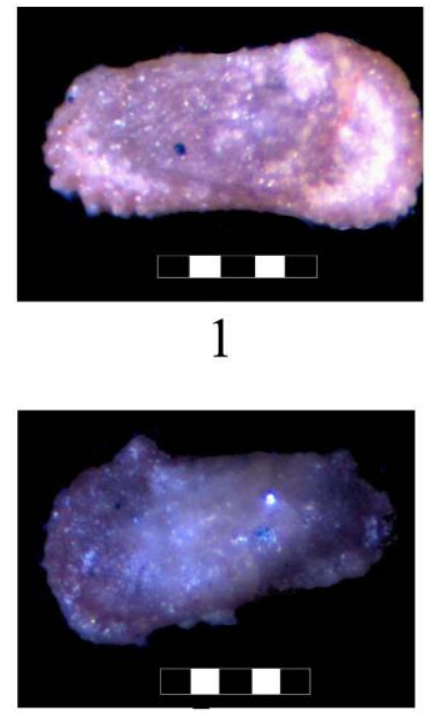

4

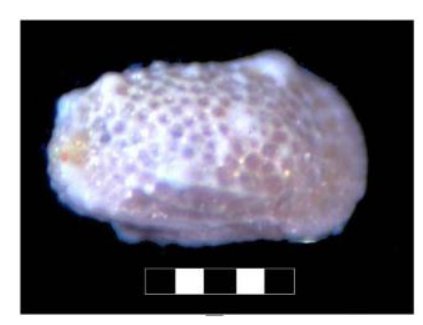

7

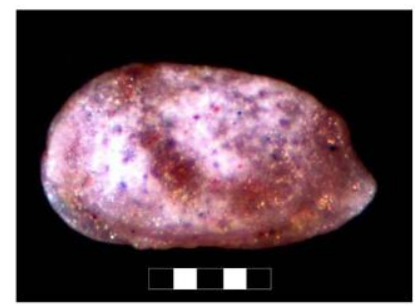

10

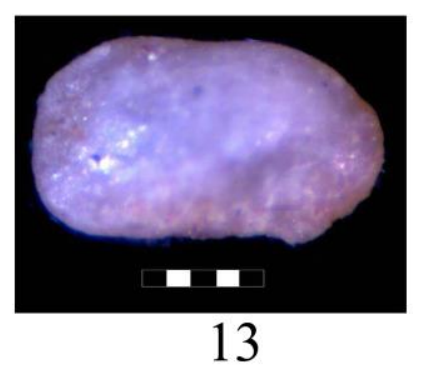

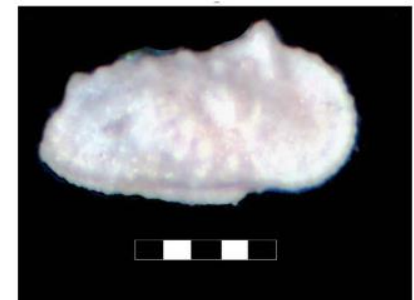

2

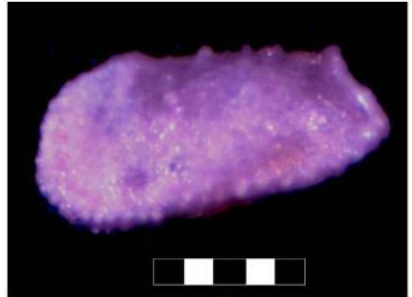

3

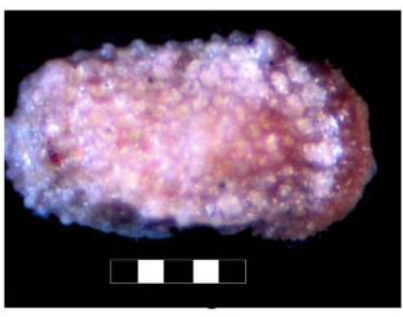

5

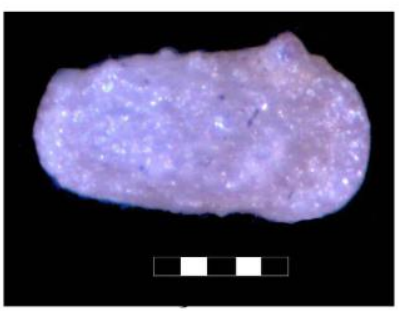

8

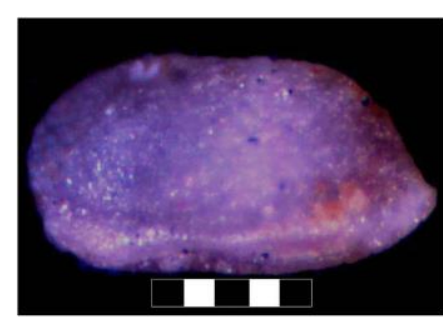

11

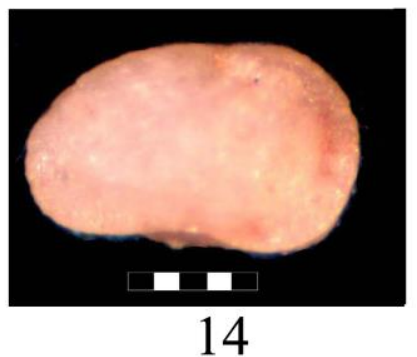

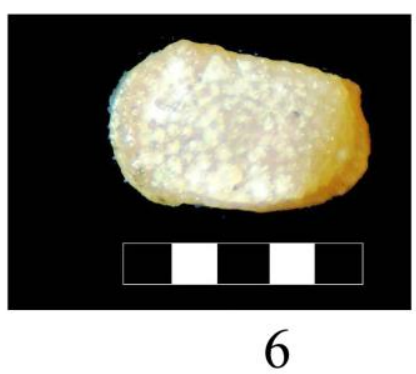

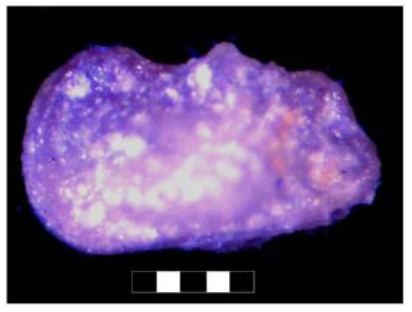

9

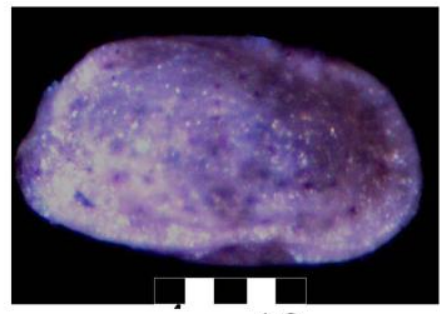

12

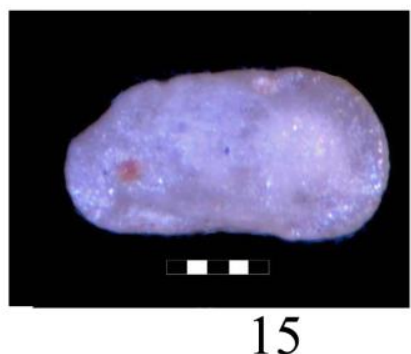


PLATE (4)
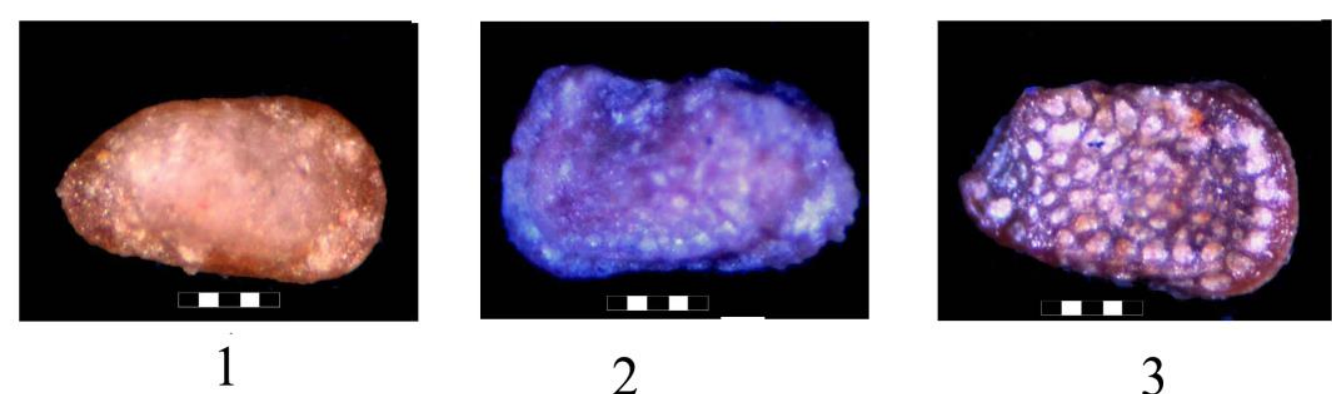

2
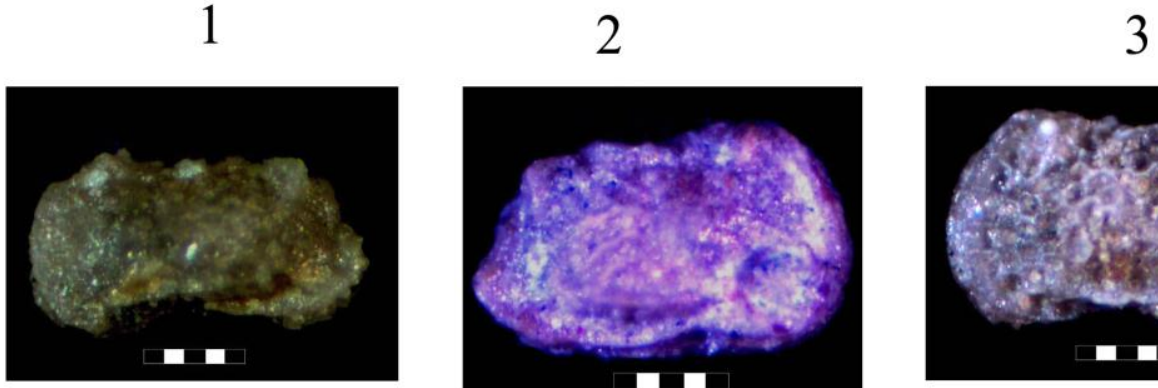

4

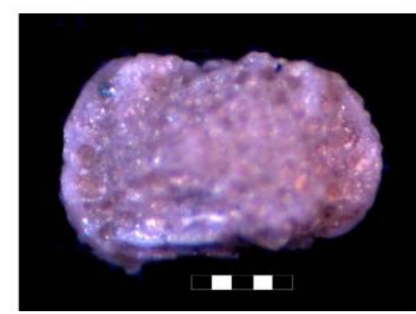

7

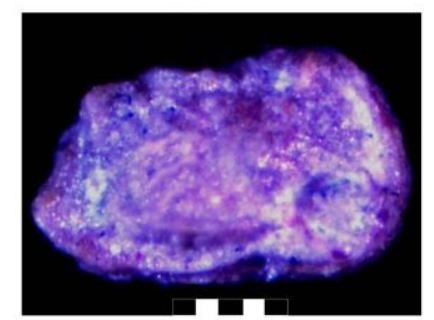

5

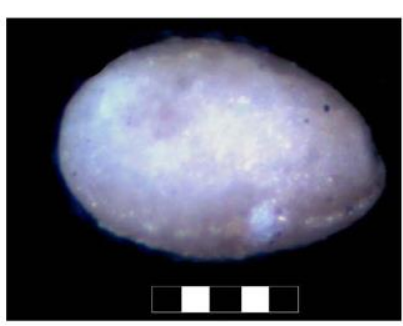

8

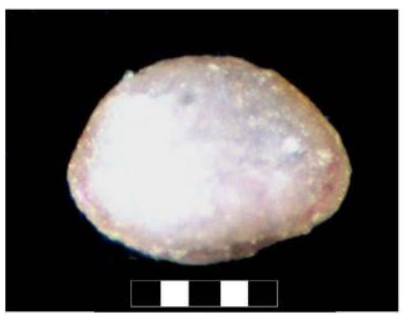

10

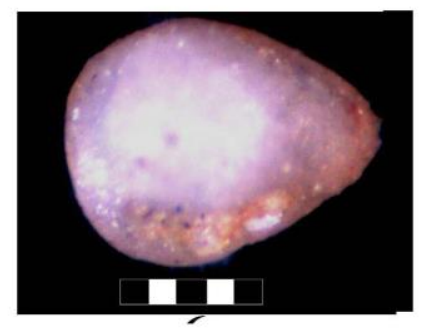

13

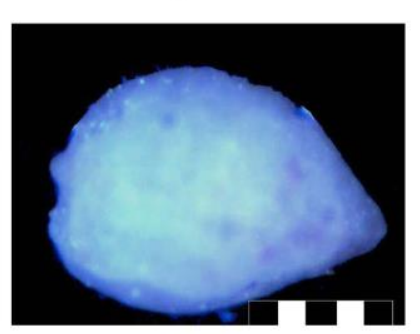

11

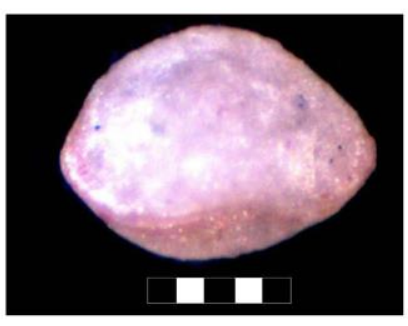

14

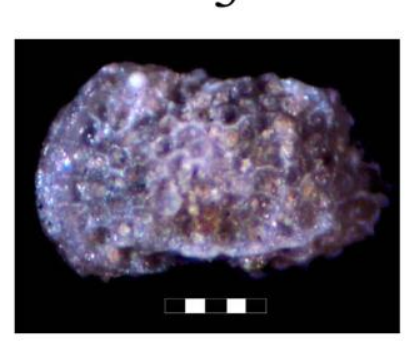

6

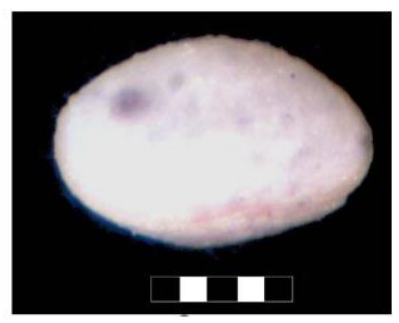

9

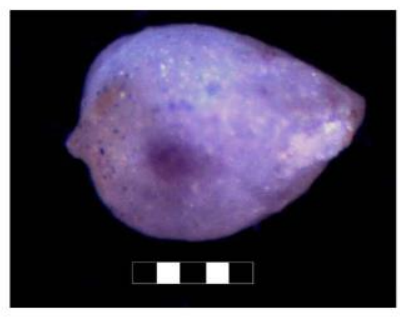

12

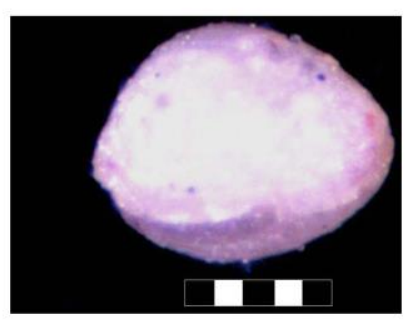

15 